Recepción: 20 / 04 / 2017

Aceptación: 20 / 05 / 2017

Publicación: 15 / 07 / 2017

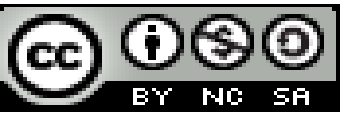

Ciencias de la Salud

Artículo de Investigación

\title{
Cáncer de mama en el hombre a propósito de un caso
}

Breast cancer in the man about a case

\section{Cancro da mama na men um relato de um caso}

Carlos H. Malatay-González ${ }^{\text {I }}$ cmalatay1@hotmail.com

Juan B. Pazmiño-Palacios II juanecopaz@gmail.com

Carlos X. Cordero-Jurado III cxcordero@gmail.com

Correspondencia: cmalatay1@ hotmail.com

I. Cirujano oncólogo del instituto Oncológico Nacional Solca; Guayaquil, Ecuador. Profesor de Cirugía de la Universidad de Guayaquil, Guayaquil, Ecuador.

Postgradista de Cirugía General del Instituto Oncológico Nacional Solca Guayaquil, Guayaquil, Ecuador.

Postgradista de Cirugía General del Instituto Oncológico Nacional Solca Guayaquil, Guayaquil, Ecuador. 


\section{Resumen}

El cáncer de mama en hombre es una enfermedad rara e infrecuente representa menos del $1 \%$ de neoplasia malignas en hombre y es responsable del $0,1 \%$ de muertes por cáncer en hombres. La edad promedio de presentación va desde los 65 a los 67 años, 10 años más tarde que en las mujeres. Su Etiología no es clara, la mayoría de las personas afectadas no tienen factores de riesgo, como son factores genéticos, hormonales y ambiéntales; su diagnostico de realiza de manera clínica, imágenes e histológica. El siguiente caso es de un paciente de 64 años que presento nódulo de $3 \mathrm{~cm}$ en mama izquierda la biopsia informa de un carcinoma ductal y se realiza mastectomía radical. El cáncer de mama masculino a menudo se diagnostica en un estadio más avanzado. El cáncer que se detecta en un estadio más avanzado tiene menos posibilidades de curarse la posibilidad de recuperación y las opciones de tratamiento. A lo mejor será porque la gente no espera que un hombre presente cáncer de mama, lo que dificulta su detección temprana. El objetivo de presentar este caso crear una mayor concientización general, los hombres aprenderán que, al igual que las mujeres, deben consultar al médico.

Palabras Clave: Cáncer; mama; hombre. 


\begin{abstract}
Breast cancer in men is a rare and uncommon disease that represents less than $1 \%$ of malignant neoplasms in men and is responsible for $0.1 \%$ of cancer deaths in men. The average age of presentation ranges from 65 to 67 years, 10 years later than in women. Its etiology is not clear, most affected people do not have risk factors, such as genetic, hormonal and environmental factors; Its diagnosis of clinical, imaging and histological. The next case is of a patient of 64 years who presented nodule of $3 \mathrm{~cm}$ in left breast. The biopsy reports a ductal carcinoma and radical mastectomy is performed. Male breast cancer is often diagnosed in a more advanced stage. Cancer that is detected in a more advanced stage is less likely to cure the possibility of recovery and treatment options. Maybe it will be because people do not expect a man to present breast cancer, making it difficult to detect them early. With the aim of presenting this case create greater general awareness, men will learn that, like women, they should consult the doctor.
\end{abstract}

Keywords: Cancer, breast, man. 


\section{Resumo}

O cancro da mama em homens é uma doença rara e pouco frequentes representa menos de $1 \%$ das neoplasias malignas em homens e é responsável por $0,1 \%$ de mortes por cancro em homens. A idade média de apresentação varia de 65 a 67 anos, 10 anos mais tarde do que as mulheres. A etiologia não é clara, a maior parte das pessoas afectadas não têm factores de risco, tais como os factores genéticos, hormonais e ambientais; faz o seu diagnóstico de clínicos, de imagem e histologicamente. O seguinte caso é uma paciente de 64 anos que presente nó 3 centímetros esquerda relatórios de biópsia da mama um carcinoma ductal e mastectomia radical é realizada. câncer de mama masculino é muitas vezes diagnosticada em um estágio mais avançado. O câncer é detectado em um estágio mais avançado é menos provável para curar a possibilidade de opções de recuperação e tratamento. Talvez seja porque as pessoas não esperam um homem cancro presente peito, tornando difícil a detecção precoce. O objetivo da apresentação deste caso criar uma maior consciência geral, os homens vão aprender que, como as mulheres, devem consultar um médico.

Palavras-chave: Cancro, peito, homem. 


\section{Introducción.}

Aunque el cáncer de mama es más común en la mujer, los hombres también pueden padecerlo, cerca del $2 \%$ de los hombres presentan con alguna sintomatología esta enfermedad. Lamentablemente, sea por condiciones sociales o por desconocimiento, estos no acuden a consulta regularmente, e incluso, los que sienten algún malestar o acostumbran a realizar chequeos periódicos generales de salud, descartan su asistencia al mastólogo o la evaluación mamaria. En promedio, desde la aparición del primer síntoma hasta el diagnóstico del cáncer de mama en el varón pueden pasar hasta 19 meses.

Al igual que en la mujer, se tienen factores de riesgo tales como la exposición a radiaciones ionizantes, el síndrome de Klinefelter, así como cualquier otra condición hiperestrogénica. Igualmente se debe revisar la historia familiar, entre el 15-20\% de los hombres con cáncer de mama tienen antecedentes familiares cercanos de esta enfermedad, e igualmente para los descendientes de un paciente masculino con cáncer de mama, debe ser tomado como antecedente relevante.

\section{Materiales y Métodos}

El trabajo se diseña como una reflexión teórica a partir de la presentación de un caso clínico del Instituto Oncológico Nacional (ION) de Ecuador. Se realizó la evaluación del paciente, quien mediante consentimiento informado asintió la publicación de los aspectos más resaltantes de su historia clínica desde el diagnóstico hasta el tratamiento definitivo de la enfermedad. 


\section{Caso Clínico.}

Paciente de 64 años de edad, diabético tipo II, casado, de Guayaquil (Ecuador) que llega por presentar nódulo de $3 \mathrm{~cm}$ de 4 años de evolución a nivel retroareolar izquierdo, duro y fijo además en región axilar izquierda se palpa ganglio menor de $1 \mathrm{~cm}$. Se solicitan examen radiológicos mamografía (nódulo de mama izquierda que radiológicamente es de aproximadamente $2.5 \mathrm{~cm}$ en región retroareolar de mama izq. Bi-rads 4) y ecografía (en mama izquierda se observa una formación nodular sólida, hipoecogénica, de márgenes microlobulados, con vascularización central y periférica, que mide aproximadamente 2.2 x $1.9 \mathrm{~cm}$, localizada hacia la región retroareolar que condiciona retracción del pezón. Piel de grosor normal bi-rads 4b de mama izquierda), donde se realiza biopsia con aguja trucut teniendo como resultado carcinoma ductal infiltrante, grado histológico 2.

Figura 1. Mamografía AP Izquierda

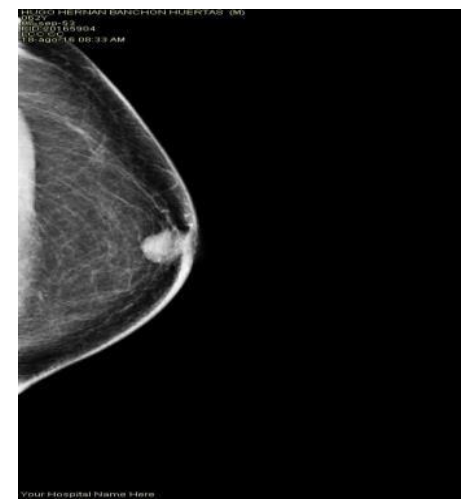

Figura 2. Mamografía oblicua Izquierda 


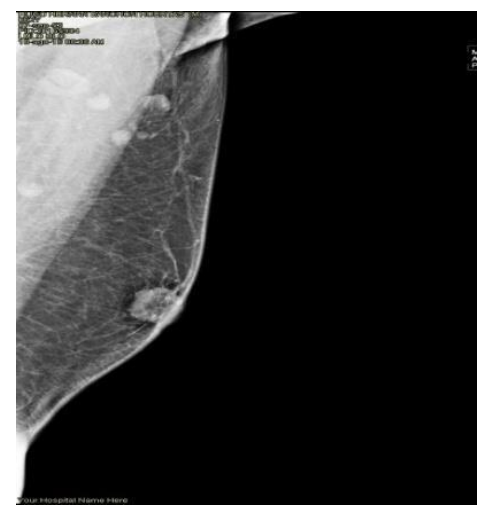

Figura 3. Ecosonografía de mama Izquierda

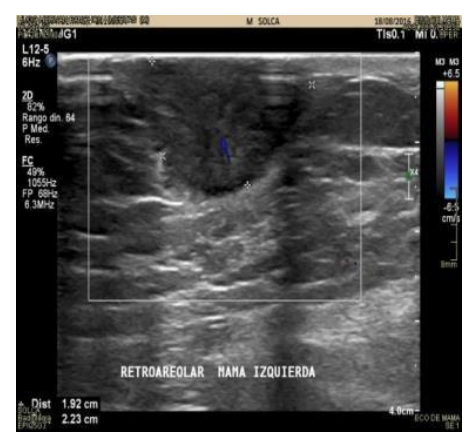

El paciente se programa para mastectomía radical cuyo resultado definitivo fue carcinoma ductal infiltrante, grado histológico 2, tamaño tumoral $3.5 \mathrm{~cm}$ invasiones: no se identifican signos de invasión linfovascular ni perineural. Piel (areola y pezón): tumor compromete la dermis profunda y superficial, sin llegar a contactar con la epidermis. Márgenes de resección: libres de neoplasia. Producto de linfadenectomía regional.- niveles I y II: ausencia de metástasis en 17 ganglios evaluados (o/17). Estatificación patológica: pt2 pno. Los Inmunohistoquimica: receptor estrogénico: positivo intenso, en el $90 \%$ de las células neoplásicas, receptor progesterona: positivo heterogéneo, en el 80\% de las células neoplásicas. el ki-67: expresión en el 15\% de las células neoplásicas . Hercep test: inmunohistoquimica: her-2 neu: negativo (1+). Al momento paciente se encuentra por parte de oncología clínica con terapia hormonal 
Figura 4. Paciente con masa en mama izquierda

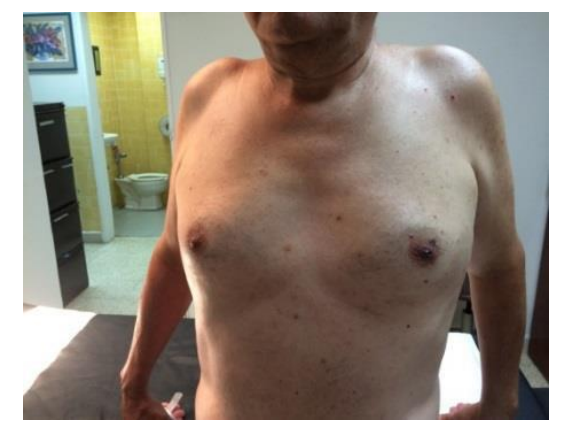

Figura 5. Paciente se realiza mastectomía izquierda

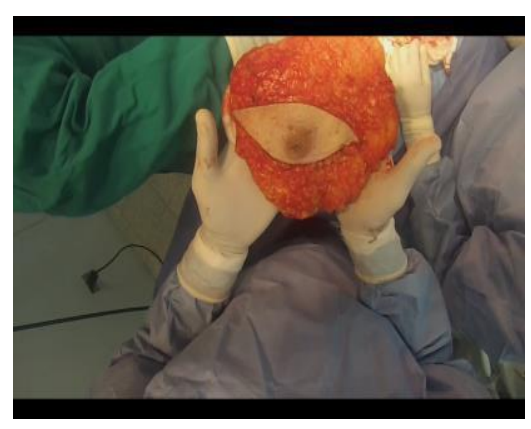

Figura 6. Paciente en controles subsecuentes con terapia hormonal

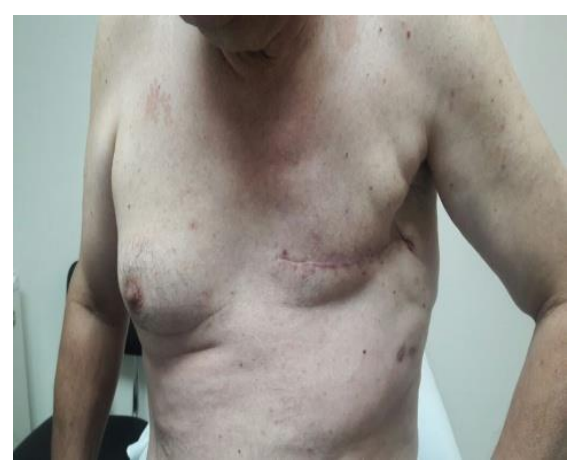

\section{Conclusiones}

La mayoría de los hombres que son diagnosticados con cáncer de mama deben someterse a algún tipo de tratamiento contra la enfermedad. El tratamiento más adecuado depende de una serie de factores, incluidos el tamaño y la ubicación del tumor, el estadio del cáncer y los 
resultados de los demás análisis de laboratorio. Las distintas alternativas en el manejo del cáncer de mama en el hombre que va desde la cirugía, cirugía del ganglio centinela en el hombre con diagnóstico de cáncer de mama, la radioterapia, la terapia hormonal y la quimioterapia. Cada una o de manera combinada se usa dependiendo de su estadio en el momento del diagnóstico. Lo importante es llegar al diagnóstico y de esta manera evitar que pase desapercibida esta patología, que no es muy común en el varón.

Desde los centros de salud y las redes de salud pública es importante realizar llamados de atención sobre la prevención y detección temprana del cáncer de mama en el varón, tal como se realiza para la mujer.

\section{Bibliografía}

Anelli A, Anelli Tfm, Youngson B, Rosen Pp, Borgen Pi. Mutations Of The Gene In Male Breast Cancer. 1995.

Bird St, Brophy Jm, Hartzema Ag, Delaney Jac, Etminan M. Male Breast Cancer And 5 $\alpha$-Reductase Inhibitors Finasteride And Dutasteride. The Journal Of Urology. 2013.

Bratman Sv, Kapp Ds, Horst Kc. Evolving Trends In The Initial Locoregional Management Of Male Breast Cancer. The Breast. 2012.

Cokmert S, Bahadir F, Guler T, Tanriverdi O. Male Breast Cancer Exhibiting Features Of Basal-Like Subtype Female Breast Cancer. Journal Of Oncological Sciences. 2016

Delgado B, Palacios L, Petit H, Barrios G, Tejada A, Pacheco C. Carcinoma De Mama En El Hombre: A Propósito De Un Caso. Revista Venezolana De Oncología. 2009.

Fentiman Is, Fourquet A, Hortobagyi Gn. Male Breast Cancer. The Lancet. 2006.

Giordano Sh. A Review Of The Diagnosis And Management Of Male Breast Cancer. The Oncologist. 2005.

Giunta G, Rossi M, Toia F, Rinaldi G, Cordova A. Male Breast Cancer: Modified Radical Mastectomy Or Breast Conservation Surgery? A Case Report And Review Of The Literature. International Journal Of Surgery Case Reports. 2017.

Golshan M, Rusby J, Dominguez F, Smith Bl. Breast Conservation For Male Breast Carcinoma. The Breast. 2007.

Gómez-Raposo C, Tévar Fz, Moyano Ms, Gómez Ml, Casado E. Male Breast Cancer. Cancer Treatment Reviews. 2010. 
Heinig J, Jackisch C, Rody A, Koch O, Buechter D, Schneider Hpg. Clinical Management Of Breast Cancer In Males: A Report Of Four Cases. European Journal Of Obstetrics \& Gynecology And Reproductive Biology. 2002.

$\mathrm{Hu}$ Sw, Chuang Jh, Tsai Kb. Immunohistochemical Expression In Male Breast Cancer: Two Case Reports. The Kaohsiung Journal Of Medical Sciences. 2006; 22.

Johansson I, Killander F, Linderholm B, Hedenfalk I. Molecular Profiling Of Male Breast Cancer--Lost In Translation? The International Journal Of Biochemistry \& Cell Biology. 2014; 53.

Johansson I, Lauss M, Holm K, Staaf J, Nilsson C, Fjällskog Ml, Et Al. Genome Methylation Patterns In Male Breast Cancer--Identification Of An Epitype With Hypermethylation Of Polycomb Target Genes. Molecular Oncology. 2015; 9.

Kamila C, Jenny B, Per H, Jonas B. How To Treat Male Breast Cancer. The Breast. 2007; 16.

Liu Jm, Chiang Yj, Chi Cl, Lo Yf, Chou Cy. Male Breast Cancer After Finasteride Therapy For Benign Prostate Hyperplasia. Urological Science. 2013; 24.

Mesa G, Matute G, Estrada M, Ocampo A, Restrepo C, Estrada J. Cáncer Mamario En Hombres. Revista Colombiana De Cirugía. 2011; 26.

Naymark P. Male Breast Cancer: Incompatible And Incomparable. Journal Of Men's Health And Gender. $2006 ; 3$.

Onami S, Ozaki M, Mortimer Je, Pal Sk. Male Breast Cancer: An Update In Diagnosis, Treatment And Molecular Profiling. Maturitas. 2010; 65.

Patel F, Achuthan R, Hyklova L, Hanby Am, Speirs V. Management Of Breast Cancer In An Asian Man With Post-Traumatic Stress Disorder: A Case Report. Journal Of Medical Case Reports. 2016; 10.

Prat A, Perou Cm. Deconstructing The Molecular Portraits Of Breast Cancer. Molecular Oncology. 2011; 5.

Sanguinetti A, Polistena A, Lucchini R, Monacelli M, Galasse S, Avenia S, Et Al. Male Breast Cancer, Clinical Presentation, Diagnosis And Treatment: Twenty Years Of Experience In Our Breast Unit. International Journal Of Surgery Case Reports. 2016; 20.

Shao T, Grossbard Ml, Klein P. Breast Cancer In Female-To-Male Transsexuals: Two Cases With A Review Of Physiology And Management. Clinical Breast Cancer. 2011; 11.

Staruch Rmt, Rouhani Mj, Ellabban M. The Surgical Management Of Male Breast Cancer: Time For An Easy Access National Reporting Database?. Annals Of Medicine And Surgery. 2016; 9.

Stranix Jt, Kwa Mj, Shapiro Rl, Speyer Jl. Invasive Micropapillary Carcinoma Of The Male Breast: Case Report And Review Of The Literature. Cancer Treatment Communications. 2015; 3.

Treves N, Holleb Ai. Cancer Of The Male Breast. A Report Of 146 Cases. Cancer. 1955; 8.

Upadhyay R, Kumar P, Sharma Dn, Haresh Kp, Gupta S, Julka Pk, Et Al. Invasive Lobular Carcinoma Of The Male Breast: A Rare Histology Of An Uncommon Disease. Journal Of The Egyptian National Cancer Institute. 2016; 28. 